\title{
Contemporary Approaches to Statistical Mechanical Probabilities: A Critical Commentary Part II: The Regularity Approach
}

\author{
Christopher J. G. Meacham \\ Published in Philosophy Compass 5 (2010): 1127-1136.
}

\begin{abstract}
This pair of articles provides a critical commentary on contemporary approaches to statistical mechanical probabilities. These articles focus on the two ways of understanding these probabilities that have received the most attention in the recent literature: the epistemic indifference approach, and the Lewis-style regularity approach. These articles describe these approaches, highlight the main points of contention, and make some attempts to advance the discussion. The second of these articles discusses the regularity approach to statistical mechanical probabilities, and describes some areas where further research is needed.
\end{abstract}

\section{Introduction}

Statistical mechanics is generally taken to account for thermodynamic phenomena, such as the melting of ice cubes in warm water, the diffusion of milk in coffee, and so on. It does this by assigning very high probabilities to the phenomena we see (ice cubes melting in warm water, milk diffusing in coffee) and very low probabilities to phenomena we don't see (broken eggs coalescing into whole ones, objects spontaneously sliding across counters).

But how should we make sense of these probabilities? Most of the discussion in the recent literature has focused on two kinds of proposals. First, there are the indifference approaches, that take statistical mechanical probabilities to be measures of rational indifference. On these views, statistical mechanical probabilities are the credences that a rational agent would have regarding a system given only certain information about what the system is like. Second, there are the regularity approaches, that take statistical mechanical probabilities to be part of an optimally simple and informative description of what the world is like. On these views, statistical mechanical probabilities are sophisticated ways of encoding the frequencies of certain kinds of events.

The merits and demerits of these two kinds of views have been subject to a lot of analysis. In this pair of articles, I will sketch the outlines of this debate, highlight the main points of contention, and make a few attempts to advance the discussion. In the previous article I provided a brief sketch of the statistical mechanics, and discussed the indifference 
approach to statistical mechanical probabilities. In this article, I'll discuss the regularity approach to statistical mechanics, and describe some outstanding issues, and some areas where further research is needed.

Due to space restrictions, my discussion will be limited in several ways. First, I will restrict my attention to the indifference and regularity approaches just described, and thus will skip over some historically important approaches to statistical mechanical probabilities. A particularly noteworthy omission is the time-average interpretation of statistical mechanical probabilities developed by Ehrenfest and Ehrenfest (1990), which is still endorsed by a number of physicists. Second, I will assume something like the Boltzmannian account of statistical mechanics proposed by Albert (2000). While this is the most widely discussed formulation of statistical mechanics in the recent literature, there are interesting alternatives. In both cases, I refer those with an interest in these issues to the more comprehensive discussions others have provided. ${ }^{1}$

\section{The Regularity Approach}

The regularity accounts of statistical mechanical probabilities that have been developed recently have taken statistical mechanical probabilities to be chances. These accounts begin by appealing to something like the regularity account of laws and chances proposed by Lewis (1994). ${ }^{2}$ On Lewis' account, the laws and chances describe certain salient regularities in the distribution of the qualitative properties. Roughly, the idea is that the laws are picked out by the set of true sentences about this distribution which best balances simplicity and informativeness. And the chances are probabilities that appear in these laws which encode information about frequencies of certain kinds of events.

More precisely, on Lewis' account, we can determine what the laws and chances of a world are in the following way. First, take a language that has only has two kinds of predicates: (a) predicates corresponding to perfectly natural properties and relations (such as mass, charge, spatiotemporal relations, etc.), and (b) a chance predicate. ${ }^{3}$ Second, take the spatiotemporal distribution of the natural properties and relations at this world, and construct every possible set of sentences that either truthfully describe some feature of the distribution, or make some chancy claim about the distribution. Third, rule out every set of sentences whose chance sentences fail to satisfy certain desiderata. ${ }^{4}$ Fourth, evaluate each set of sentences with respect to three criteria: (a) simplicity, (b) informativeness, and (c) fit (where 'fit' is a measure of how high a chance the chance sentences in this set assign to the actual history of the world), and select the set of sentences that, on balance, best fits these three desiderata. The laws of this world consist of every proposition expressing a regularity that can be derived from this optimal set. And the chances of this world are the

\footnotetext{
${ }^{1}$ See Sklar (1993), Uffink (2006), Frigg (2008b), and the references provided therein.

${ }^{2}$ Regularity accounts of statistical mechanical probabilities more or less along these lines have been proposed by Loewer (2001), Hoefer (2007), Winsberg (2008), Albert (2009) and Frigg and Hoefer (2010).

${ }^{3}$ On Lewis' account the chance predicate will be a 4-place predicate, where the first slot takes a proposition, the second slot a number, the third slot a time and the fourth slot a world. See Lewis (1983) for a discussion of the distinction between the natural and non-natural properties.

${ }^{4}$ I.e., the resulting chances must all be assigned to the world in question (the fourth slot in the chance predicates must be filled by this world), the resulting chances must satisfy the probability axioms (the values of the first and second slots in the chance predicates must together satisfy the appropriate constraints), and so on.
} 
chances entailed by these laws. ${ }^{5}$

Before we proceed, it's worth noting why it's important to specify the language in which these sentences are formulated. We need a fixed language in order to make sensible assessments of the simplicity of sets of sentences. As Lewis (1983) notes, without this restriction, this scheme would lead to absurd results. E.g., pick an arbitrary world $w$, construct a language which contains the predicate $P_{w}$, where $P_{w}$ applies to all and only the contents of worlds qualitatively identical to this one, and consider the singleton set containing the sentence $\forall x P_{w}(x)$. This sentence will yield a maximally informative and simple description of this world, and since this sentence entails every true regularity, every true regularity will be a law. This entails that it's impossible for there to be accidental regularities, which is absurd.

The regularity approach to statistical mechanical probabilities has been subject to several criticisms. Some of these criticisms concern the regularity approach as a whole, and have little to do with attempting to provide a regularity account of statistical mechanical probabilities per se. Although these general objections raise important issues, I won't consider them in detail here (though I will flag some of these more general worries in section 3). Instead, I'll focus on objections specific to the regularity account of statistical mechanical probabilities.

The two most commonly pressed objections are (i) statistical mechanical probabilities aren't the right kinds of things to be the chances of the regularity theory, and (ii) even if the regularity theory did yield chances of this kind, these chances would have values that differ from the statistical mechanical probabilities. Let's look at each of these criticisms in turn.

\subsection{Objection 1: Statistical Mechanical Probabilities Aren't the Right Kinds of Things to Be the Regularity Theory's Chances}

The first objection to the regularity approach is that statistical mechanical probabilities aren't the right kinds of things to be the chances that the regularity theory posits. This objection has been pushed in a couple of different ways.

First, many have noted that Lewis would not have allowed statistical mechanical probabilities to be chances. Lewis (1986) held that non-trivial chances are incompatible with deterministic dynamical laws. Since statistical mechanical probabilities are compatible with deterministic laws, statistical mechanical probabilities can't be chances.

However, as Loewer (2001) points out out, there is nothing about Lewis' rationale that requires this restriction. Indeed, the opposite is the case-Lewis' rationale for why the laws can sometimes be chancy provides a straightforward justification for statistical mechanical chances. ${ }^{6}$ Lewis' idea was that the chances give us a simple way to say something informative about certain patterns of events that resist a simple, comprehensive description. Even though we can't concisely state when (say) $A$-type events will be followed by $B$-type events, we can note that roughly $30 \%$ of the $A$-type events are followed by $B$-type events.

\footnotetext{
${ }^{5}$ Two questions. First, are the laws propositions or interpreted sentences? Lewis (1983) was explicitly agnostic on this issue; I assume they are propositions here. Second, what if our evaluation of the sets of sentences yields ties - several sets of sentences which satisfy these desiderata equally well? In this case, Lewis (1983) suggests that we form a new set of sentences containing only the sentences that appear in all of the sets that are tied, and that we use this new set to derive the laws.

${ }^{6}$ See Loewer (2001), Hoefer (2007), Winsberg (2008) and Albert (2009).
} 
And given that the pattern of $A$ and $B$-type events resists a simple description, these kinds of statements may best satisfy, on balance, the goals of simplicity and informativeness that we're striving for (where fit can be understood as a kind of informativeness).

But this rationale applies straightforwardly to deterministic probabilities. Consider a set of sentences describing deterministic dynamics. Adding a sentence describing the initial conditions will make this set much more informative-together, these sentences will provide a complete description of the world. But in most cases, adding such a sentence will also make the set far too complicated to provide the optimal balance between simplicity and informativeness. Chances give us a way to take the middle road here-we can add some probabilistic claims to the deterministic dynamics that are both simple and informative. And the set that results from adding such sentences could well provide the optimal balance between simplicity and informativeness.

Another objection along similar lines is that Lewis (1994) restricts the laws to propositions that express regularities. But a law assigning statistical mechanical probabilities to initial conditions does not seem to be expressing a regularity. ${ }^{7}$ However, as Winsberg (2008) notes, there is nothing about Lewis' rationale that requires this restriction. Indeed, the opposite is the case-given the motivating idea that the laws are picked out by the sentences that provide the optimally balanced simple and informative description of the world, the restriction to propositions that express regularities looks ad hoc.

Second, some have pushed the objection that statistical mechanical probabilities can't be the chances of the regularity theory by arguing that they can't be chances simpliciter. This is generally done by arguing that statistical mechanical probabilities fail to satisfy certain intuitions we have about chances, and then concluding that statistical mechanical probabilities don't fit our ordinary conception of chance well enough to deserve the name. ${ }^{8}$

These kinds of considerations are relevant to the question of whether statistical mechanical probabilities are chances. With respect to the issues we're concerned with, however, this objection is irrelevant. The question we're interested in is whether the regularity approach can provide a viable account of statistical mechanical probabilities. Whether we call these probabilities "chances" or not, and whether they conform to our ordinary use of the word "chance", is besides the point.

Third, some have objected that statistical mechanical probabilities can't be the chances of the regularity theory, because this would leave them unable to play certain roles that we want statistical mechanical probabilities to play. In particular, it seems statistical mechanical probabilities place constraints on rational belief. If statistical mechanical probabilities were the chances of the regularity theory, then they would need to place constraints on rational credences via something like Lewis' Principal Principle. ${ }^{9}$ But, it's argued, statistical mechanical probabilities can't impose the right constraints on belief via the Principal Principle. Therefore, statistical mechanical probabilities can't be the chances of the regularity theory. ${ }^{10}$

Although it's true that statistical mechanical probabilities can't impose the right constraints on belief via Lewis' Principal Principle, this is largely an artifact of Lewis' formulation. Lewis characterized the Principal Principle with dynamical chances in mind-

\footnotetext{
${ }^{7}$ Though it's not clear that this problem is particular to statistical mechanical probabilities, since it's not clear that any claim about chances is 'expressing a regularity'.

${ }^{8}$ See Schaffer (2007).

${ }^{9}$ See Lewis (1986).

${ }^{10}$ See Schaffer (2007).
} 
fundamental chances that describe how likely it is that the world will transition from one state into another-and as a result, his formulation of the principle builds in various assumptions regarding what chances are like. But if we remove these eccentric features of Lewis' original principle, we get a principle that applies equally well to both dynamical and non-dynamical chances. ${ }^{11}$

To sum up, the objection that statistical mechanical probabilities can't be the regularity theory's chances doesn't hold up under scrutiny. Although it's true that Lewis' regularity account could not accommodate statistical mechanical chances, these incompatibilities arise from inessential features of his account that are easily removed. So these worries do not pose a threat to the regularity understanding of statistical mechanical probabilities.

\subsection{Objection 2: The Chances of the Regularity Approach Will Have Values that Don't Match the Statistical Mechanical Proba- bilities}

The second objection to the regularity approach concerns whether this approach could yield chances with the right values to be the statistical mechanical probabilities. By and large, these objections have focused on Lewis' account, and have taken the following form. If a Lewis-style regularity account of statistical mechanical probabilities is correct, then the set of sentences that best balances the desiderata of simplicity, informativeness and fit will be the set stating the classical statistical mechanical laws. ${ }^{12}$ But the optimal set with respect to these desiderata won't be the set stating the classical statistical mechanical laws. Therefore a Lewis-style regularity account of statistical mechanical probabilities cannot be correct.

The key to this objection is the claim that the set stating the classical statistical mechanical laws won't be the best candidate with respect to these desiderata. People have argued for this conclusion in a number of ways.

One way of pursuing this argument points to the fact that, on the formulations of the classical statistical mechanical laws that are currently the most popular, the Past Hypothesis will be one of the laws. The Past Hypothesis claims that the initial state of the universe satisfies some particular low entropy macrostate. And a description of this macrostate in a language with only perfectly natural vocabulary would seem to be very complicated. Thus it seems the set stating the classical mechanical laws will also be very complicated, and so will not be the optimal set. ${ }^{13}$

A second way of pushing this objection argues that sets stating other statistical theories will do better than the set stating the classical statistical mechanical laws in terms of

\footnotetext{
${ }^{11}$ A number of people have suggested ways of reformulating the Principal Principle in a statistical mechanicsfriendly way. Perhaps the most straightforward reformulation has been independently suggested by Arntzenius (1995), Meacham (2005) and Nelson (2009); further arguments in favor of this formulation have been given by Meacham (2010). Other statistical mechanics-friendly reformulations of the Principal Principle have been suggested by Loewer (2001), Hoefer (2007) and Ismael (2010).

${ }^{12}$ Strictly speaking, this doesn't need to be the case. The optimal set might just contain sentences from which the classical statistical mechanical laws are derivable. And if so, some of the criticisms that follow may not get off the ground. However, the proponents of the regularity approach have also generally assumed that the sentences in the optimal set will state something like the laws of classical statistical mechanics. So I'll grant this assumption in what follows.

${ }^{13}$ See Schaffer (2007) and Winsberg (2008).
} 
Lewis' third desiderata, fit. Consider, for example, a theory whose probabilistic assignments to the initial conditions can be graphed as a bell curve centered on the actual initial conditions, instead of the flat assignment provided by classical statistical mechanics. It's suggested that by making the curve steep enough, the gain in fit will override any minor increase in complexity that positing the bell curve introduces. ${ }^{14}$ Thus the sets positing these probabilities will turn out to be better candidates than the set stating the classical statistical mechanical laws.

A third and similar way of pushing this objection argues that the set stating the dynamical laws and the precise initial conditions will be a better candidate than the set stating the classical statistical mechanical laws, with any loss in simplicity being outweighed by a gain in informativeness. ${ }^{15}$

In response to this objection, a number of people have noted that while some of these worries might be problems for Lewis' original account, they are not problems for all regularity approaches. And there are natural ways of modifying Lewis' account to get around these difficulties.

Here is one way of doing this, suggested by Barry Loewer. ${ }^{16}$ Loewer proposes three modifications to Lewis' account. First, instead of evaluating every set of true sentences in a privileged language, we evaluate every set of true sentences in every language. Second, we add a fourth criterion, salience, to our list of desiderata, where the salience of a set of sentences hangs on how salient, helpful and accessible a description of the world they provide. Alternatively, instead of adding a fourth criterion, we can require that Lewis' three desiderata be understood in terms of salience: how much salient information the set of sentences provides, how simple and graspable this information is in salient respects, and so on. Third, allow any proposition derivable from the optimal set to be a law, not just those that express regularities. ${ }^{17}$

This approach has the benefit of not requiring us to posit a distinction between the perfectly natural properties and the rest. ${ }^{18}$ And, more importantly, this proposal gets around the kinds of objections sketched above, which rely on notions of simplicity and informativeness that are at odds with the kinds of things that are salient and helpful to beings like us.

The first worry was that the Past Hypothesis would be very complicated when expressed in the language of perfectly natural properties, and so the set stating the classical statistical mechanical laws could not be the best candidate. But on Loewer's account this worry evaporates, since the sentences don't need to be formulated in the language of perfectly natural properties.

The second worry was that a set that posits a probabilistic bell curve around the ac-

\footnotetext{
${ }^{14}$ See Frigg (2008a).

${ }^{15}$ See Schaffer (2007) and Frigg (2008a).

${ }^{16}$ Barry Loewer sketched this proposal at the "Descrying the World in Physics" workshop at the Central European University in 2006. (This way of fleshing out Loewer's proposal comes from Eddon and Meacham (2006).) Similar-but more radically relativized—ways of modifying Lewis' account have been proposed by Hoefer (2007), Winsberg (2008), Callender and Cohen (2009) and Frigg and Hoefer (2010).

${ }^{17}$ This yields the somewhat odd consequence that propositions expressing tautologies can count as laws. This worry also arises for Lewis' original proposal, since regularities like $\forall x(x=x)$ will qualify as laws. In either case, if this bothers us, we can simply restrict the laws to the non-tautological propositions entailed by the optimal set.

${ }^{18}$ Though how much of a benefit this turns out to be depends on how much pressure there is from other debates to adopt such a distinction. See Lewis (1983) for arguments in favor of positing such a distinction, and see Eddon and Meacham (2006) and Callender and Cohen (2009) for some replies.
} 
tual initial conditions will be a better candidate than the set stating the classical statistical mechanical laws. But again, this does not seem to be a worry for Loewer's proposal. A salient and accessible specification of a probabilistic bell curve over the actual initial conditions (in the canonical parameterization of the phase space) requires a specification of where in the phase space the actual initial conditions are. And doing that in a salient and accessible way is no easier than providing a salient and accessible characterization of the initial conditions. We can, of course, re-parameterize the phase space in order to make this bell curve a uniform distribution over possible states, making the probability distribution easy to characterize in a salient and accessible way. But then it is our specification of the desired parameterization that becomes difficult to specify in a salient and accessible way. Either way, one can argue that the set stating the classical statistical mechanical laws will provide a more salient and useful description of the world.

The third worry was that the set stating the dynamical laws and the precise initial conditions is a better candidate than the set stating the classical statistical mechanical laws. On Loewer's account this is not a worry, since a set which includes a precise specification of the initial conditions would not be more informative, accessible or useful in salient respects to beings like ourselves than the set stating the classical statistical mechanical laws.

Loewer's modification of Lewis' account gets around these three worries, but it also raises a fourth worry. We can see, at least roughly, what it means for a set of sentences to be more salient, helpful or explanatory for people like us. But it is hard to see how one's standards of salience could be entirely objective, or even objective at all. Consider again the language containing the predicate which applies to all and only those things belonging to this world, $P$, and the singleton set containing the sentence ' $\forall x(P x)$ '. We can see why this set does poorly with regards to salience for people like us: we don't find it helpful, explanatory, or salient to our interests or the way we think about the world. But it's hard to see how to go about evaluating salience in objective terms. So it seems that by adding salience into the mix, Loewer's characterization risks making the laws of nature relative to us in some sense. ${ }^{19}$

But upon closer examination, it becomes clear that Loewer's proposal is no more 'usdependent' than Lewis' original account. To see this, recall the complaints that were raised by van Fraassen (1989) and others about Lewis' original proposal. The complaint was that it's hard to see how the standards of informativeness and simplicity could be entirely objective. And if these standards aren't objective, then it seems the resulting laws won't be objective either. Thus, it was argued, Lewis' reliance on notions like simplicity and informativeness risked making the laws of nature relative to us in some sense.

Lewis' initial suggestion for how to get around these worries was to use our current standards of simplicity and informativeness and then to rigidify them. This gives us a grasp on what the standards of simplicity and informativeness are. And since we've rigidified these standards, what the laws are won't depend on things like whether or not there are sentient beings around, or what they're like. What the laws are is an objective, agentindependent matter. ${ }^{20}$

\footnotetext{
${ }^{19}$ This worry has been raised regarding approaches like this one by Frigg (2008a) and Winsberg (2008).

${ }^{20}$ To get better grip on what the proposal is, consider the following hypothetical objection: "Different agents will have different standards of simplicity and informativeness. Indeed, different temporal stages of the same agents may have different standards of simplicity and informativeness. So on this account, there will be different laws for different person stages. That's absurd."
} 
Loewer can make precisely the same response to the us-dependence worries described above. Namely, that we should evaluate salience with respect to beings like ourselves, and then rigidify these standards. Again, this will yield an account of laws in which what the laws are is an objective, agent-independent matter.

Lewis (1994) later backed away from this appeal to rigidification, worrying that rather than getting rid of the problem, it just makes it harder to state. But irrespective of one's feelings regarding this worry, appealing to salience doesn't make the regularity approach any more us-dependent. If one thinks that appealing to rigidification gets rid of us-dependence, then neither Loewer nor Lewis is subject to the worry. If one thinks that rigidification fails to get rid of the worry, then both Lewis and Loewer will need to say something else. Either way, the same kinds of moves will be available to them both. ${ }^{21}$

The literature has produced several arguments for the conclusion that Lewis' account won't yield chances with the right values to be the statistical mechanical probabilities. While some of these objections may succeed against Lewis' account, they do not pose a threat to the general viability of the regularity approach, since there are ways of modifying Lewis' account to circumvent these difficulties.

That said, we should be careful to appreciate the tentative nature of this conclusion. First, as the worries raised for Lewis' account show, problems which are hard to see in the abstract can pop into view when we try to fill in the details. And since little has yet been done to fill in these details, further worries may arise when things are fleshed out more carefully. Second, there are more generic worries-regarding how to understand the notion of 'fit', for example—which I've been ignoring. ${ }^{22}$ So a final assessment will have

Here is how the proponent of rigidification replies: “There aren't different laws for different person stages. There's one set of laws for everyone, at all times. These are the laws that happen to be picked out by your current standards. Now, I grant that different agents at different times will have different standards. And I grant that if they were reading this passage, they would get the misleading impression that it's their standards that correctly pick out the facts about what the laws are. But they'd be wrong. Their standards aren't the right ones. Your standards are the right ones. And it's those standards that determine the objective facts about the laws."

${ }^{21}$ One might try to resist this claim by holding that desiderata like simplicity and informativeness differ from salience in important ways. For example, one might argue that while we can find some seemingly objective judgments with respect to simplicity and informativeness - that the function $f(x)=x$ is objectively simpler than the function $f(x)=x^{3}+5 x^{1 / 2}+9$, say-the same is not true for salience. But this position is hard to maintain, since we can find seemingly objective judgments regarding salience as well. Consider the following question: which of the following string of ten digits-2, 2, 2, 2, 2, 2, 8, 2, 2, 2-is the salient digit? One could argue that this question has an objectively right answer: 8 .

One could grant this point and still argue that salience is problematic. That is, one could argue that even if one can find some seemingly objective judgments regarding salience, there are too few of these judgments to yield the robust and discriminating notion of salience that Loewer's proposal requires. But the same can be said with respect to informativeness and simplicity. Consider informativeness. The only straightforward judgments regarding informativeness stem from this claim: if one set of statements entails another, then the first set is at least as informative as the second. But these kinds of judgments alone are far too weak to yield the robust and discriminating notion of informativeness that Lewis' account requires.

It has been suggested in conversation that there might be another way in which desiderata like simplicity and informativeness differ from salience. In particular, it has been suggested that the notion of salience is contextdependent, while the notions of simplicity and informativeness are not. However, this position seems hard to maintain, since simplicity and informativeness seem just as context-dependent as salience. One can maintain, for example, that what's salient in the context of statistical mechanics differs from what's salient in the context of biology. But it is just as plausible to maintain that what's informative in the context of statistical mechanics differs from what's informative in the context of biology.

${ }^{22}$ See Elga (2004). 
to wait until more has been done to fill in the details of the account.

\section{Future Research}

Let me conclude by sketching two areas for further research.

First, a number of key issues in this debate hang on how we should understand statistical mechanical explanations. One objection to the indifference approach to statistical mechanical probabilities is that it can't recover statistical mechanical explanations. As was shown in part 1, this explanatory objection appears to pose a severe challenge to the viability of the indifference approach.

The regularity approach to statistical mechanical probabilities is not immune to explanatory worries either. In particular, this approach is susceptible to the same kinds of explanatory objections as the regularity accounts of laws. When applied to regularity accounts of laws, the worry is this. In order to explain why regularities obtain, we usually appeal to the laws. But on the regularity approach, the laws are just certain kinds of regularities. So on the regularity approach to laws, it looks like these explanations become circular-we're trying to use regularities to explain themselves. ${ }^{23}$ And a similar complaint could be raised against regularity approach to statistical mechanical probabilities.

In both cases, whether these worries turn out to be decisive objections or not hangs on details regarding the correct account of explanation, and in particular, the correct account of statistical mechanical explanation. Unfortunately, few of those working on explanation have made a serious effort to assess the kinds of explanations that statistical mechanics seems to provide. ${ }^{24}$ As a result, the literature on explanation is in a poor state to resolve these issues. So further work in this area is badly needed.

A second area for future research is exploring whether there are viable ways of making sense of statistical mechanical probabilities other than the two mainstream approaches discussed here. The explanatory worries facing the indifference approach have convinced many that this approach is untenable. At the same time, there is a substantial literature documenting the counterintuitive implications of regularity accounts of laws and chances. ${ }^{25}$ And these worries have convinced many that regularity approaches are untenable as well. This leaves those who feel both worries in a bind with respect to how to make sense of statistical mechanical probabilities.

One option is to mitigate these worries by pairing a regularity account of statistical mechanical probabilities with a non-regularity account of laws. ${ }^{26}$ Another option is to try to work out a non-epistemic and non-regularity account of statistical mechanical probabilities. ${ }^{27}$ This might be something like a "propensity" account of chance, shorn of the usual dynamical assumptions. Or it might be something that bears little resemblance to the accounts of chance currently on offer. In any case, further work exploring these options would be welcome. ${ }^{28}$

\footnotetext{
${ }^{23}$ See Armstrong (1983) and van Fraassen (1989).

${ }^{24}$ With Strevens (2009) being an exception.

${ }^{25}$ For example, see van Fraassen (1989), Carroll (1994), Maudlin (2007) and Hall (2010). See Loewer (2004) and Hall (2010) for replies to some of these objections.

${ }^{26}$ Hoefer (2007) and Winsberg (2008) both defend views which explicitly allow for this possibility.

${ }^{27}$ See Ismael (2009) for a proposal of this kind.

${ }^{28}$ I would like to thank Phil Bricker, Maya Eddon, Barry Loewer and an anonymous referee for comments and discussion.
} 


\section{References}

Albert, David. 2000. Time and Chance. Harvard University Press.

Albert, David. 2009. "Physics and Chance.” Unpublished Manuscript.

Armstrong, David. 1983. What is a law of nature. Cambridge University Press.

Arntzenius, Frank. 1995. "Chance and the Principal Principle: Things Ain't What They Used To Be.” Unpublished Manuscript.

Callender, Craig and Jonathan Cohen. 2009. "A Better Best System Account of Lawhood." Philosophical Studies 145:1-34.

Carroll, John. 1994. Laws of Nature. Cambridge University Press.

Eddon, Maya and Christopher Meacham. 2006. "No Work For a Theory of Universals." Unpublished Manuscript.

Ehrenfest, Paul and Tatiana Ehrenfest. 1990. The conceptual foundations of the statistical approach in mechanics. Dover.

Elga, Adam. 2004. "Defeating Dr. Evil with self-locating belief." Philosophy and Phenomenological Research 69:383-396.

Frigg, Roman. 2008a. "Chance in Boltzmannian Statistical Mechanics.” Philosophy of Science 75:670-681.

Frigg, Roman. 2008b. A Field Guide to Recent Work on the Foundations of Statistical Mechanics. In The Ashgate Companion to Contemporary Philosophy of Physics, ed. Dean Rickles.

Frigg, Roman and Carl Hoefer. 2010. Determinism and Chance from a Humean Perspective. In The Present Situation in the Philosophy of Science.

Hall, Ned. 2010. "Humean reductionism about laws of nature." Unpublished Manuscript.

Hoefer, Carl. 2007. "The Third Way on Objective Probability: A Skeptic's Guide to Objective Chance." Mind 116:549-596.

Ismael, Jenann. 2009. "Probability in Deterministic Physics." The Journal of Philosophy 106:89-108.

Ismael, Jenann. 2010. “A Modest Proposal About Chance.” Forthcoming in The Journal of Philosophy.

Lewis, David. 1983. "New Work for a Theory of Universals." Australasian Journal of Philosophy 61:343-377.

Lewis, David. 1986. A Subjectivist's Guide to Objective Chance. In Philosophical Papers, Vol. 2. Oxford University Press pp. 83-132.

Lewis, David. 1994. “Humean Supervenience Debugged.” Mind 103:473-490. 
Loewer, Barry. 2001. "Determinism and Chance." Studies in the History of Modern Physics 32:609-620.

Loewer, Barry. 2004. Humean Supervenience. In Readings on Laws of Nature, ed. John Carroll.

Maudlin, Tim. 2007. The Metaphysics Within Physics. Oxford University Press.

Meacham, Christopher J G. 2005. "Three Proposals Regarding a Theory of Chance." Philosophical Perspectives 19:281-307.

Meacham, Christopher J G. 2010. "Two Mistakes Regarding the Principal Principle." British Journal for the Philosophy of Science .

Nelson, Kevin. 2009. “On Background: Using Two-Argument Chance.” Synthese 1:165186.

Schaffer, Jonathan. 2007. "Deterministic Chance?" The British Journal for the Philosophy of Science 58:113-140.

Sklar, Lawrence. 1993. Physics and Chance. Cambridge University Press.

Strevens, Michael. 2009. Depth: An Account of Scientific Explanation. Harvard University Press.

Uffink, Jos. 2006. Compendium to the Foundations of Classical Statistical Physics. In Handbook for the Philosophy of Science: Philosophy of Physics, ed. Jeremy Butterfield. Elsevier pp. 924-1074.

van Fraassen, Bas. 1989. Laws and Symmetry. Oxford University Press.

Winsberg, Eric. 2008. "Laws and Chances in Statistical Mechanics." Studies in History and Philosophy of Modern Physics 39:872-888. 